\title{
Transcriptional activities of bovine lactoferrin (LTF) gene promoter haplotypes
}

\section{B Bahar ${ }^{1}$, F O'Halloran $^{2}$, L Giblin $^{2}$, T Sweeney ${ }^{1}$}

${ }^{1}$ School of Agri. Food Science \& Veterinary Medicine, University College Dublin, Belfield, Dublin, Ireland

${ }^{2}$ Moorepark Food Research Centre, Teagasc, Fermoy, Cork, Ireland

Email:bojlul.bahar@ucd.ie

Introduction Bovine lactoferrin is an important innate immune protein with a wide range of host defense functions including anti-microbial and anti-viral properties. Genetic polymorphisms present in the promoter region of the lactoferrin $(L T F)$ gene have the potential to affect gene expression (Teng 2002). Once characterized, such polymorphisms can be used for the identification of designer herds which produce increased levels of lactoferrin protein in milk. The objectives of this study were to identify major LTF promoter haplotypes and to investigate the in vitro transcriptional activities of the $L T F$ promoter haplotypes.

Material and methods The experimental population comprised of Holstein-Friesians ( $\mathrm{n}=47$ ), Jersey $(\mathrm{n}=6)$, Jersey $X$ Friesian cross $(\mathrm{n}=5)$, Norwegian Red $(\mathrm{n}=7)$, Montbéliard $(\mathrm{n}=4)$, Norwegian Red X Friesian cross $(\mathrm{n}=1)$ and Bos indicus $(\mathrm{n}=8)$. A total of 31 single nucleotide polymorphisms (SNPs), previously identified through sequencing of $2.2 \mathrm{~kb}$ bovine LTF promoter $(\mathrm{n}=80)$ (O'Halloran et al., 2009), were used to deduce haplotypes. To identify promoter haplotypes, the locus wise genotype data of SNPs were analysed. Haplotype analysis was performed using EM algorithm in the Arlequin software 3.11. Haplotype frequencies were estimated considering the abundance of an individual haplotype over the total haplotypes in the population $(\mathrm{n}=156)$. Two bovine LTF haplotypes (BtLTF_H1a and BtLTF_H2a) were chosen for the in vitro promoter assay. Promoter region (stretching from $-2200 \mathrm{bp}$ to $+1 \mathrm{bp}$ relative to the transcription start site) of seven animals (four representing the haplotype BtLTF_H1a and three representing BtLTF_H2a) were cloned into a pGL4.17 luciferase expression vector system (Promege Corp.). The basal transcriptional activities of the LTF promoters were evaluated in vitro using a mouse mammary epithelial cell line (NMuMG). The data of the normalized luciferase activity (promoter assay) is compared by ' $\mathrm{t}$ ' test and presented as mean \pm standard error.

Results A total of 51 unique haplotypes were identified in the study population of which 8 haplotypes represented $>70 \%$ of the population (Figure 1). In silico analysis of the $2.2 \mathrm{~kb}$ promoter revealed two major haplotypes (BtLTF_H1a and BtLTF_H2a) that differed at ten SNP loci that altered putative transcription factors binding sites for both constitutive (at $28,-1702$ ) and inducible (at $-131,-270,-586,-2047,-2077,-2122,-2140$ and -2151) expression. Transfection studies demonstrated that these two haplotypes differed significantly $(\mathrm{P}<0.001)$ in their basal promoter transcriptional activity (Figure 2). Cows with the BtLTF_H1a haplotype were expected to have greater lactoferrin protein concentration in milk compared to herdmates with the BtLTF_H2a haplotype.
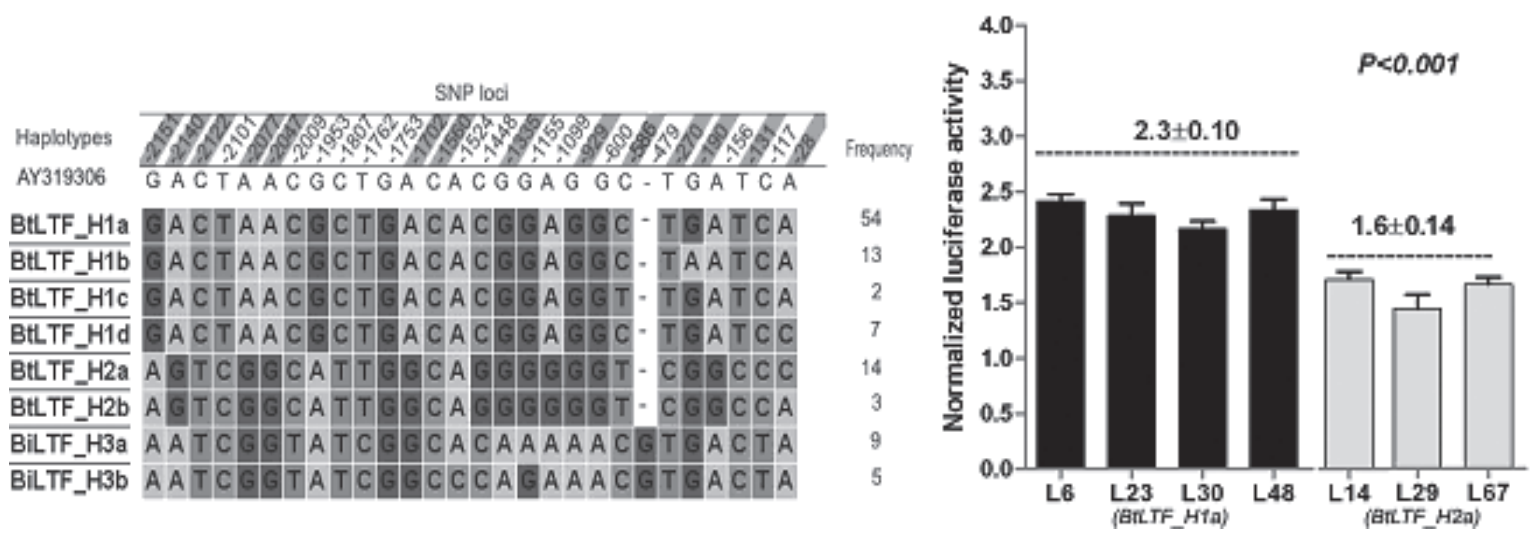

Figure 1 Major promoter haplotypes of the bovine $L T F$ gene $(2.2 \mathrm{~kb})$

Figure 2 In vitro basal activities of the bovine $L T F$ promoter haplotypes.

Conclusions Two predominant LTF promoter haplotypes were identified (BtLTF_H1a and BtLTF_H2a). Transfection studies in mammary epithelial cells showed that promoter constructs of the BtLTF_H1a haplotype had increased transcriptional activity in vitro compared to constructs of the BtLTF_H2a haplotype. A LTF haplotype based selection strategy in breeding may aid in increasing lactoferrin protein concentration in the bovine milk to promote and maintain health and well being in the dairy cow and the consumer. It may also facilitate identification of designer herds for production of milk with high content of lactoferrin.

Acknowledgements This research was supported by the Department of Agriculture, Food and Fisheries, Govt. of Ireland under the Food Institutional Research Measure (FIRM).

\section{Reference}

Teng, C.T. 2002. Biochemistry and Cell Biology. 80, 7-16.

O’Halloran, F., Bahar, B., Buckley, F., O’Sullivan, O., Sweeney, T. and Giblin, L. 2009. Biochimie. 91, 68-75. 